\title{
THE FINITE INTERSECTION PRINCIPLE AND GENERICITY
}

\author{
DAVID DIAMONDSTONE, ROD DOWNEY, NOAM GREENBERG, AND DAN TURETSKY
}

\begin{abstract}
We show that a $\Delta_{2}^{0}$ Turing degree computes solutions to all computable instances of the finite intersection principle if and only if it computes a 1-generic degree. We also investigate finite and infinite variants of the principle.
\end{abstract}

\section{INTRODUCTION}

The axiom of choice has always occupied a central place in mathematical logic and in applications of logic to the rest of mathematics. Classically, the axiom of choice has many equivalent forms. These forms, however do not remain equivalent when examined through the microscope of either computability theory or of reverse mathematics. In [DM13] Dzhafarov and Mummert examined a sequence of choicelike principles concerning the existence of maximal subfamilies with intersection properties. Their motivation was to investigate the strength of analogues of the axiom of choice within the framework of second order arithmetic. Among the principles they introduced was the finite intersection principle, which says that every (necessarily countable) collection of sets of natural numbers has a subcollection, maximal with respect to the property that the intersection of finitely many sets in the subcollection have nonempty intersection. They also investigated variants stating that the intersection of $n$ many sets in the subcollection is nonempty, for a fixed natural number $n \geqslant 2$. They showed that these intersection principles are related to other mathematical principles such as the atomic model theorem (from model theory) and the existence of cohesive sets (from computability theory).

Some of the complexity of these concepts arises from the fact that second order arithmetic does not admit abstract sets. A collection $\mathcal{A}$ of subsets of $\omega$ has to come with an enumeration: a sequence of sets $\left\langle A_{m}\right\rangle$ such that $\left\{A_{m}: m<\omega\right\}=\mathcal{A}$. We allow repetitions in the sequence. Of course there are many ways to enumerate a family of sets and different enumerations will usually not be computationally equivalent.

Nonetheless below we use notation which blurs the difference between families and their enumerations. For example, if $\mathcal{A}$ and $\mathcal{B}$ are sequences of sets then we write $\mathcal{B} \subseteq \mathcal{A}$ to denote that every element of the sequence $\mathcal{B}$ also appears somewhere in $\mathcal{A}$, but possibly in a different location.

Definition 1.1. An instance of an intersection problem (or simply a family of sets) is a sequence $\mathcal{A}=\left\langle A_{m}: m<\omega\right\rangle$ of subsets of $\omega$. A FIP-solution for $\mathcal{A}$ is an sequence $\mathcal{B}$ of subsets of $\omega$ such that $\mathcal{B} \subseteq \mathcal{A}$ and the intersection of any finitely

Downey was supported by the Marsden Fund. Greenberg was supported by the Marsden Fund and by a Rutherford Discovery Fellowship from the Royal Society of New Zealand. Diamondstone and Turetsky were supported by the Marsden Fund via postdoctoral scholarships. 
many sets from $\mathcal{B}$ is nonempty. For $n \geqslant 2$, a $n$ IP-solution ${ }^{1}$ for $\mathcal{A}$ is a sequence $\mathcal{B}$ of subsets of $\omega$ such that $\mathcal{B} \subseteq \mathcal{A}$ and the intersection of any $n$ many sets from $\mathcal{B}$ is nonempty. A solution is maximal if it is not properly contained in any other solution for $\mathcal{A}$.

This paper investigates these principles from the point of view of computability theory. We thus ignore the effects of restricted induction by concentrating on $\omega$ models, in other words on the behaviour in the Turing degrees. We thus define:

Definition 1.2. A FIP-degree is a Turing degree which computes a maximal FIPsolution for any computable family. Similarly we define $n$ IP-degrees for each $n \geqslant 2$.

In [DM13] the authors showed that a FIP-degree is also a $n$ IP-degree for each $n \geqslant 2$, and that any $(n+1)$ IP-degree is also a $n$ IP-degree. They showed that every 2IP-degree is hyperimmune (not $\mathbf{0}$-dominated). They also showed that the degrees in the following collections are all FIP-degrees:

- nonzero computably enumerable (c.e.) degrees;

- degrees which are not $\mathbf{0}^{\prime}$-dominated;

- degrees that compute generics which meet any prescribed sequence of dense $\Pi_{1}^{0}$ sets of binary strings.

A property common to all degrees in the collections above is that they compute 1-generic sets. Indeed this is sufficient. We show:

Theorem 1.3. Every 1-generic degree is a FIP-degree.

On the other hand there is no known example of a FIP or even a 2IP degree which does not compute a 1-generic set. So it is natural to ask:

(1) Are the FIP degrees the same as those which compute 1-generic sets?

(2) Are the FIP degrees and the 2IP degrees the same?

While these questions remain open, in this paper we answer them in the affirmative for the case of $\Delta_{2}^{0}$ degrees (degrees computable from the halting problem).

Theorem 1.4. A $\Delta_{2}^{0}$ degree is FIP if and only if it is 2IP if and only if it computes a 1-generic set.

Theorem 1.3 will be proved in Section 2; Theorem 1.4 will be proved in Section 3. In Section 4 we investigate variants in which we require elements of the collections to be finite, or intersections to be infinite.

1.1. Preliminaries. Let $\mathcal{A}=\left\langle A_{m}: m<\omega\right\rangle$ be a computable instance of an intersection problem. An index-set of a solution $\mathcal{B}$ is a set $B$ of numbers such that $\mathcal{B}=\left\{A_{m}: m \in B\right\}$. A Turing degree which can enumerate an index-set $B$ can compute some enumeration of the indexed solution $\mathcal{B}$. Technically the converse does not hold, but as is noticed in [DM13], when one constructs families one can ensure that the converse does hold. When we build a family, into every nonempty set in that family we insert a unique identifying marker - some element $i \in \omega$ which we do not include in any other set of the family. All sets in a solution are nonempty, so for any set in the solution, we can search it until we find an identifying marker, at which point we know the set's index in the original family. Hence any solution $\mathcal{B}$ for the family we build has a unique index-set $B$, and any degree which computes $\mathcal{B}$

\footnotetext{
${ }^{1}$ The notation for the corresponding principle in [DM13] is $\bar{D}_{2} I P$.
} 
can enumerate $B$. We can then identify a solution $\mathcal{B}$ with an enumeration of its index-set $B$.

We also note that the discovery of intersections is a c.e. event. Conversely, for every c.e. family $\mathcal{A}$ we can construct a computable family $\hat{\mathcal{A}}$ such that the indexsets of solutions for $\mathcal{A}$ are precisely the index-sets of solutions for $\hat{\mathcal{A}}$. Initially let each $\hat{A}_{m}$ be empty. When we notice that $\bigcap_{m \in F} A_{m} \neq \varnothing$ for some finite set $F$, enumerate some large element into $\bigcap_{m \in F} \hat{A}_{m}$. Since the elements enumerated are large, $\hat{\mathcal{A}}$ is computable.

For that reason it does not matter if the instances and solutions are uniformly computable or uniformly c.e.: a degree $\mathbf{d}$ is a FIP-degree if and only if for every c.e. family $\mathcal{A}$ there is a maximal FIP-solution for $\mathcal{A}$ which is $\mathbf{d}$-c.e.

In the main construction below we will use the recursion theorem to force our opponent to reveal, during the construction, a maximal solution for a family that we are building. For this to work we need to show that the opponent can compute solutions uniformly in indices for families. This is done by constructing a universal (and acceptable) family.

Proposition 1.5. Both FIP and 2IP have acceptable universal families. That is, there is a computable family $\mathcal{U}$ such that every maximal FIP-solution for $\mathcal{U}$ has FIPdegree. Furthermore, from a computable index for a family $\mathcal{A}$ one can effectively find an index for a Turing functional $\Phi$ such that for any maximal FIP-solution $\mathcal{Q}$ for $\mathcal{U}, \Phi(\mathcal{Q})$ is a maximal FIP-solution for $\mathcal{A}$. The same holds for 2IP.

Proof. Let $\left\langle\mathcal{A}_{m}\right\rangle$ be an effective enumeration of all uniformly c.e. families, where $\mathcal{A}_{m}=\left\langle A_{m, n}\right\rangle_{n<\omega}$. Enumerate a family $\mathcal{U}=\left\langle U_{m, n}\right\rangle_{m, n<\omega}$ satisfying: (1) every nonempty $U_{m, n}$ has a unique identifying marker; and (2) for all finite $F \subset \omega^{2}$, $\bigcap_{(m, n) \in F} U_{m, n} \neq \varnothing$ if and only if for all $m, \bigcap_{n \in F^{[m]}} A_{m, n} \neq \varnothing$. As discussed above every solution for $\mathcal{U}$ enumerates its index-set. If $Q$ is an index-set for a maximal FIP-solution for $\mathcal{A}$ then for all $m, Q^{[m]}=\{n:(m, n) \in Q\}$ is an index-set for a maximal FIP-solution for $\mathcal{A}_{m}$. The proof for 2IP is similar.

\section{Genericity IMPLIES FIP}

In [DM13] the authors implicitly describe a natural notion of forcing which approximates an enumeration of an index-set of a maximal FIP-solution for a given instance. Let $\mathcal{A}$ be a computable family. We let $\mathbb{P}_{\mathcal{A}}$ be the collection of finite strings $\sigma$ whose range is an index-set of a FIP-solution for $\mathcal{A}$. That is, strings $\sigma$ such that $\bigcap_{m<|\sigma|} A_{\sigma(m)}$ is nonempty. Equivalently we can think of the conditions in $\mathbb{P}_{\mathcal{A}}$ as solutions of finite length. The set of conditions $\mathbb{P}_{\mathcal{A}}$ is c.e.; it is ordered by extension. If $G \subset \mathbb{P}_{\mathcal{A}}$ is a filter then $f_{G}=\bigcup G$ is an enumeration of an index-set of a solution.

Let $(\mathbb{Q}, \leqslant \mathbb{Q})$ be a c.e. partial ordering of a c.e. set $\mathbb{Q}$. Recall that a filter $G \subset \mathbb{Q}$ is 1-generic for $\mathbb{Q}$ if for all c.e. sets of conditions $D \subseteq \mathbb{Q}$ the filter $G$ meets or avoids $D$ : either $G \cap D$ is nonempty or some condition in $G$ has no extension in $D$ (for more see for example [GM03]). The usual notion of 1-genericity is 1-genericity for Cohen forcing $2^{<\omega}$; equivalently for $\omega^{<\omega}$ since the latter can be effectively densely embedded into the former.

Lemma 2.1. The solution given by a 1 -generic filter $G \subseteq \mathbb{P}_{\mathcal{A}}$ is maximal. 
Proof. Suppose that $A_{n}$ intersects $\bigcap_{m \in K} A_{m}$ for all finite $K \subset$ range $f_{G}$. Then the collection of conditions $\sigma \in \mathbb{P}_{\mathcal{A}}$ whose range contains $n$ is dense around $G$, and is c.e. Since $G$ is 1 -generic it meets this collection of conditions and so the solution it indexes contains $A_{n}$.

Thus, any degree which can enumerate a 1-generic filter for $\mathbb{P}_{\mathcal{A}}$ can compute a maximal solution for $\mathcal{A}$. We show that any 1-generic set (in the Cohen sense) does. The point is that provided that $\mathcal{A}$ does not have a finite maximal solution, there is an effective isomorphism $i$ from $\omega^{<\omega}$ to $\mathbb{P}_{\mathcal{A}}$. We can define it by recursion, starting by mapping the empty string to itself. Let $\sigma \in \omega^{<\omega}$ and suppose that $i(\sigma) \in \mathbb{P}_{\mathcal{A}}$ is defined (and has length $|\sigma|$ ). Since $\mathcal{A}$ does not have a finite maximal solution, $i(\sigma)$ has infinitely many one-bit extensions in $\mathbb{P}_{\mathcal{A}}$ : if only finitely many sets in $\mathcal{A}$ intersect $\bigcap_{m<|\sigma|} A_{i(\sigma)(m)}$ then $\left\{A_{i(\sigma)(m)}: m<|\sigma|\right\}$ can be extended to a finite maximal solution for $\mathcal{A}$. The one-bit extensions of $i(\sigma)$ can be enumerated effectively; we let $i\left(\sigma^{\wedge} k\right)$ be the $k^{\text {th }}$ one on the list.

If $G \subset \omega^{<\omega}$ is 1 -generic then $i[G]$ is a 1 -generic filter for $\mathbb{P}_{\mathcal{A}} ; i[G]$ is c.e. in $G$. Hence $G$ can enumerate an index-set for a maximal FIP-solution for $\mathcal{A}$, and so can compute such a maximal solution. This proves Theorem 1.3.

\section{2IP SOMETIMES IMPLIES GENERICITY}

To prove Theorem 1.4 it remains to show that every $\Delta_{2}^{0}$ 2IP degree computes a 1-generic set. It is easier to show that every $\Delta_{2}^{0}$ FIP degree computes a 1-generic set; we will give the simplified proof first and then elaborate on the argument to get the full result. We first motivate the construction by considering hyperimmune degrees (which are the degrees which compute weakly 1-generic sets).

3.1. Hyperimmunity. As mentioned above, in [DM13] the authors showed that every 2IP-degree is hyperimmune. We give a simplified proof of this fact.

We enumerate a family $\mathcal{X}=\left\langle X_{n}\right\rangle$ of sets (as mentioned above we can make these sets uniformly computable rather than merely uniformly c.e.) Every nonempty set in $\mathcal{X}$ will contain a unique identifier, implying that given a solution (or even an enumeration of a solution) we can enumerate the index-set for the solution.

There are two kinds of sets in $\mathcal{X}$. We let $\{b(e): e<\omega\} \cup\{a(e, k): e \leqslant k<\omega\}$ be an enumeration of $\omega$. The set $X_{b(e)}$ will be a marker which gives us evidence toward believing that $\varphi_{e}$ is total. The set $X_{a(e, k)}$ will indicate that $\varphi_{e}(k) \downarrow$. The following rules determine the family:

(1) $X_{b(e)}$ is nonempty if and only if $\varphi_{e}(e) \downarrow$.

(2) $X_{a(e, k)}$ is always nonempty. It intersects $X_{b(e)}$ if and only if $X_{b(e)}$ is already nonempty (by rule (1)), and further $\varphi_{e}(k) \downarrow$.

(3) These are the only restrictions on intersection. If $e \neq e^{\prime}$ then every nonempty set with an $e$ index $\left(X_{b(e)}\right.$ or $\left.X_{a(e, k)}\right)$ intersects every nonempty set with an $e^{\prime}$ index. Every $X_{a(e, k)}$ and $X_{a\left(e, k^{\prime}\right)}$ intersect as well.

Let $\mathbf{d}$ be a 2IP-degree; let $Q$ be a $\mathbf{d}$-c.e. index-set of a maximal 2IP-solution for $\mathcal{X}$. There are many nonempty sets in $\mathcal{X}$, and so $Q$ only contains indices of nonempty sets. For each $e$, each $X_{a(e, k)}$ intersects all nonempty elements of $\mathcal{X}$ except possibly for $X_{b(e)}$. Hence, if $b(e) \notin Q$ then $a(e, k) \in Q$ for all $k \geqslant e$. If $b(e) \in Q$ then $a(e, k) \in Q$ if and only if $\varphi_{e}(k) \downarrow$.

We define a d-computable function $g$ which will escape each computable function. Given an input $k$ we will try to define $g(k)$ to escape $\varphi_{e}(k)$ for all $e \leqslant k$. The 
question of course is which of these actually converge. We consult $Q$. Using d, enumerate $Q$ up to a stage $s=s(k)>s(k-1)$ such that for all $e \leqslant k$, either $b(e) \in$ $Q_{s}$ or $a(e, k+1) \in Q_{s}$ (or both). Now there are two cases. For $e=k$, we check if $b(k) \in Q_{s}$; if so we know that $\varphi_{k}(k) \downarrow$ and so we can define $g(k)$ to escape $\varphi_{k}(k)$. For $e<k$, we check if both $b(e)$ and $a(e, k)$ are in $Q_{s}$; if so then we know that $\varphi_{e}(k) \downarrow$ and so we can define $g(k)$ to escape $\varphi_{e}(k)$. If these conditions don't hold for $e$ then we give up on trying to escape $\varphi_{e}(k)$.

To show that $g$ is not majorised by any computable function, suppose that $\varphi_{e}$ is total. Then $X_{b(e)}$ intersects every nonempty set in $\mathcal{X}$ and so necessarily $b(e) \in Q$; similarly $a(e, k) \in Q$ for all $k \geqslant e$. Let $s$ be the stage at which $b(e)$ is enumerated into $Q$. There are two possibilities. If no $a(e, k)$ is in $Q_{s}$ then $s(e) \geqslant s$ and so $g$ escapes $\varphi_{e}$ on input $e$. Otherwise let $k$ be the greatest such that $a(e, k) \in Q_{s}$. Then $s(k) \geqslant s$ (recall that to find $s(k)$ we search for either $b(e)$ or $a(e, k+1)$ ) and so $g$ escapes $\varphi_{e}$ on input $k$.

3.2. Genericity and $\Delta_{2}^{0}$ FIP degrees. We prove that every $\Delta_{2}^{0}$ FIP-degree computes a 1-generic set.

3.2.1. Discussion. We enumerate a family $\mathcal{X}=\left\langle X_{n}\right\rangle$ of sets. Naïvely, we aim to define a monotone map $\Gamma$, mapping finite pieces $\mathcal{B}$ of solutions for $\mathcal{X}$ to binary strings, and extend it continuously with the aim that on a maximal solution $\mathcal{Q}$ the map will produce a 1-generic set. The very basic and imprecise idea is as follows. We want to meet or avoid the $e^{\text {th }}$ c.e. set of strings $W_{e}$. To this we devote one of the sets $X_{b(e)}$. We see a finite solution $\mathcal{B} \subset \mathcal{X}$ such that $\Gamma(\mathcal{B})$ is defined, has not met $W_{e}$ yet, but also has not avoided $W_{e}$ yet: it has an extension $\rho$ in $W_{e}$. We intersect $\bigcap \mathcal{B}$ with $X_{b(e)}$, ensuring that $\mathcal{B} \cup\left\{X_{b(e)}\right\}$ is a solution as well, which we map to $\rho$. If $\mathcal{Q}$ is a maximal solution and $W_{e}$ is dense around $\Gamma(\mathcal{Q})$ then $X_{b(e)}$ will intersect all finite pieces of $\mathcal{Q}$ and so by maximality $X_{b(e)} \in \mathcal{Q}$. Ideally this would mean that some finite piece of $\mathcal{Q}$ which contains $X_{b(e)}$ is mapped to a string in $W_{e}$, and so $\Gamma(\mathcal{Q})$ meets $W_{e}$.

We can think of this process dynamically, working with a particular $\Delta_{2}^{0}$ solution $\mathcal{Q}$ : As we see strings in $W_{e}$ extend longer and longer initial segments of $\Gamma(\mathcal{Q})$, we intersect $X_{b(e)}$ with more and more of $\mathcal{Q}$; since eventually $X_{b(e)} \in \mathcal{Q}$ our opponent will have to present $X_{b(e)}$ at some finite stage. To do this the opponent needs to change their current approximation to $\mathcal{Q}$ and in that way "give us permission" to map $\Gamma(\mathcal{Q})$ to extend some string in $W_{e}$.

This does not quite work, as we now discuss.

Sets vs. sequences. As mentioned above, a solution $\mathcal{Q}$ for $\mathcal{X}$ can be identified with an enumeration of its index-set $Q$. Rather than mapping initial segments of solutions to strings, we actually will map finite subsets of its index-set to strings. The functional $\Gamma$ will forget the order. The reason for this, essentially, is that the family $\mathcal{X}$ is determined by its intersections and these do not notice order. More specifically, suppose that $\mathcal{B}_{0}$ and $\mathcal{B}_{1}$ are two finite solutions which enumerate the same set. Suppose that we define $\Gamma\left(\mathcal{B}_{0}\right)$ and $\Gamma\left(\mathcal{B}_{1}\right)$ to be distinct. We then see an extension $\rho$ of $\Gamma\left(\mathcal{B}_{0}\right)$ in $W_{e}$, intersect $X_{b(e)}$ with $\bigcap \mathcal{B}_{0}$ and map $\mathcal{B}_{0}{ }^{\wedge} X_{b(e)}$ to $\rho$. This intersection means that $\mathcal{B}_{1}^{\wedge} X_{b(e)}$ is also an initial segment of a solution, but it is possible that currently we do not see a string in $W_{e}$ extending $\Gamma\left(\mathcal{B}_{1}\right)$, so we cannot now define $\Gamma\left(\mathcal{B}_{1}^{\wedge} X_{b(e)}\right)$ to be a string in $W_{e}$. We have to define it somehow, since it is a legitimate solution and we do not know if an extension in $W_{e}$ will ever appear. 
Once we did this, the set $X_{b(e)}$ is useless to us when we try to force $\Gamma(\mathcal{Q})$ for maximal extensions $\mathcal{Q}$ of $\mathcal{B}_{1}{ }^{\wedge} X_{b(e)}$ into $W_{e}$. Dynamically thinking, $X_{b(e)}$ already appears in the presented solution and keeping intersecting it with sets in the solution will not cause a change and a permission.

Thus, $\Gamma$ is defined on finite index-sets of solutions.

Witnesses. Even so, the plan above requires elaboration. The role of $X_{b(e)}$ is to translate a $\Pi_{2}^{0}$ fact $-W_{e}$ being dense around $\Gamma(Q)$ - to a $\Sigma_{1}^{0}$ event, the appearance of $b(e)$ in $Q$. This is similar to the hyperimmune construction above, where the set $X_{b(e)}$ was used to translate the $\Pi_{2}^{0}$ fact, namely the totality of $\varphi_{e}$, to a c.e. event. To make use of this we need to adjoin to $b(e)$ a witness (or blocker).

In detail, we explain why we cannot map any finite index-set to a string in $W_{e}$ simply on the merit of containing $b(e)$. Suppose that the solution which is presented to us at stage $s$ is $Q_{s}=\{0,1,2, \ldots, s\}$. Say that we defined $\Gamma(\{0,1, \ldots, k\})=\sigma_{k}$, and that gradually we discover extensions $\rho_{k}$ of $\sigma_{k}$ in $W_{e}$. The plan is to gradually intersect $X_{b(e)}$ with $\bigcap X_{i}$, and for $Q$ to change as to contain $b(e)$. However the opponent may place $b(e)$ in various locations. For example, after intersecting $X_{b(e)}$ with $X_{0} \cap X_{1}$ the opponent presents the solution $\{0,1, b(e)\}$ and we map that solution to $\rho_{1}$. The opponent then returns to the solution $\{0,1,2,3, \ldots\}$ and we intersect $b(e)$ with $X_{0} \cap X_{1} \cap X_{2} \cap X_{3}$ (as the strings $\rho_{2}$ and $\rho_{3}$ are revealed). Then the opponent presents the solution $\{0,1,2,3, b(e)\}$. However we cannot map this solution to $\rho_{3}$ since $\rho_{3}$ may not extend $\rho_{1}$, but $\{0,1, b(e)\} \subset\{0,1,2,3, b(e)\}$.

This is why we use sets with $a$-type indices. Roughly, in the scenario above, a special set $X_{a(e,\{0,1\})}$ will be also intersected with $X_{0} \cap X_{1} \cap X_{b(e)}$, and we wait for the appearance of both $b(e)$ and $a(e,\{0,1\})$ to define a new $\Gamma$ computation mapping to $\rho_{1}$. The set $X_{a(e,\{0,1\})}$ will not intersect the set $X_{a(e,\{0,1,2,3\})}$ so no solution contains both. We can then map the solution $\{0,1, b(e), a(e,\{0,1\})\}$ to $\rho_{1}$ and $\{0,1,2,3, b(e), a(e,\{0,1,2,3\})\}$ to $\rho_{3}$ without violating the monotony of $\Gamma$.

Solutions and paths. For simplicity, to ensure that $\Gamma$ is monotone we define it on a tree of finite sets. Sets will be added to the domain of $\Gamma$ by the following rule:

(*) At stage $s$ we may select a set $E \in \operatorname{dom} \Gamma$, some $e \leqslant s$ such that $b(e) \notin E$ and some $\rho \in W_{e, s}$ which properly extends $\Gamma(E)$. We then let $D=E \cup$ $\{b(e), a(e, E)\}$. We ensure that $D$ is an index-set of a solution for $\mathcal{X}_{s+1}$ (by enumerating an element into $\left.\bigcap_{m \in D} X_{m}\right)$ and let $\Gamma(D)=\rho$.

We are not intending to take every action permitted by this rule; rather, in preparation for the construction, we are promising that all actions we do take will obey this rule. There will be many selections permitted by this rule which we do not take.

From each $D \in \operatorname{dom} \Gamma$ we can read off how it was created; its predecessor is the $\subseteq$-greatest $E$ such that $a(e, E) \in D$ for some $e$. So we can recover the sequence $e_{0}, e_{1}, \ldots$ of indices which were used (the order of $e$ 's for which $b(e)$ were added to $D)$. On the other hand this sequence of $e$ 's completely determines the set $D$. Notationally it would be more convenient to track these sequences of $e$ 's. Thus, during the construction we enumerate a tree $T \subseteq \omega^{<\omega}$ consisting of injective strings of numbers. We index the sets in $\mathcal{X}$ by $b(e)$ for $e<\omega$ and $a(\tau)$ for $\tau \in \omega^{<\omega} ; X_{a(\tau)}$ will be nonempty if and only if $\tau \in T$. We then define

$$
D(\tau)=\{b(e): e \in \text { range } \tau\} \cup\{a(\sigma): \sigma \preccurlyeq \tau\} .
$$


In the notation of the above rule, $a(\tau)=a_{\tau(|\tau|-1), D\left(\left.\tau\right|_{|\tau|-1}\right)}$. The map $\tau \mapsto D(\tau)$ is a bijection, and so for simplicity we define $\Gamma$ on $T$. As usual we let $\mathcal{D}(\tau)=\left\{X_{m}\right.$ : $m \in D(\tau)\}$.

The entire construction will be determined by the enumerations into $T$ and the definition of $\Gamma$. At the beginning of stage $s$ we already have $T_{s}$ and have enumerated the family $\mathcal{X}_{s}=\left\langle X_{n, s}\right\rangle_{n<\omega}$. If $\tau$ is a string which is added to $T$ at stage $s\left(\tau \in T_{s+1}-T_{s}\right)$ then we enumerate a large number into $\bigcap \mathcal{D}(\tau)$ at that stage. These and the unique identifiers for nonempty sets are the only enumerations we make into $\mathcal{X}$. We can reformulate the principle above using this language:

(*) At stage $s$ we may select a string $\tau \in T_{s}$, some $e \notin \operatorname{range} \tau$ such that $\tau^{\wedge} e \notin T_{s}$ and some $\rho \in W_{e, s}$ which properly extends $\Gamma(\tau)$. We then enumerate $\tau^{\wedge} e$ into $T_{s+1}$ and let $\Gamma(\tau)=\rho$.

We start with $T_{0}$ consisting only of the empty string, mapped by $\Gamma$ to the empty string. For $s<\omega$ the tree $T_{s}$ is finite and so $\mathcal{X}_{s}$ contains only finitely many nonempty sets. Since $T$ is a tree it is easy to see that $\Gamma$ is strictly monotonic: if $\tau \prec \tau^{\prime}$ then $\Gamma(\tau) \prec \Gamma\left(\tau^{\prime}\right)$. Inductively we see that if $\tau \in T$ and $e \in$ range $\tau$ then $\Gamma(\tau)$ extends some string in $W_{e}$.

For compactness of presentation we let $T_{\omega}=T$ and $\mathcal{X}_{\omega}=\mathcal{X}$. Let $s \leqslant \omega$ (so $s$ is either finite or is $\omega$ ). If $\tau$ and $\tau^{\prime}$ are incompatible strings on $T_{s}$ then we never intersect $X_{a(\tau)}$ and $X_{a\left(\tau^{\prime}\right)}$. On the other hand if $\tau<\tau^{\prime}$ then $D(\tau) \subseteq D\left(\tau^{\prime}\right)$. Let $\tau \prec \tau^{\prime} \in T_{s}$, let $\mathcal{D} \subset \mathcal{X}_{s}$ be finite and suppose that $X_{a\left(\tau^{\prime}\right), s}$ intersects $\bigcap \mathcal{D}$. Numbers entering $X_{a\left(\tau^{\prime}\right)}$ come from enumerations of extensions of $\tau^{\prime}$ into $T$. Hence $X_{a(\tau), s}$ also intersects $\bigcap \mathcal{D}$. Note also that $X_{a(\diamond>), s}$ is nonempty and that it intersect $\bigcap \mathcal{F}$ for any finite solution $\mathcal{F} \subseteq \mathcal{X}_{s}$.

Let $Q_{s}$ be an index-set of a maximal FIP-solution for $\mathcal{X}_{s}$. The discussion above shows that: (1) $a(\langle\rangle) \in Q_{s} ;(2)$ if $a(\tau), a\left(\tau^{\prime}\right) \in Q_{s}$ then $\tau$ and $\tau^{\prime}$ are comparable; and (3) if $a\left(\tau^{\prime}\right) \in Q_{s}$ and $\tau<\tau^{\prime}$ then $a(\tau) \in Q_{s}$. That is, the set of strings $\tau \in T_{s}$ such that $a(\tau) \in Q_{s}$ is a nonempty but possibly finite path in $T_{s}$. We call that path $f_{s}$ (if it is finite then $f_{s}$ is the longest $\tau$ such that $\left.a(\tau) \in Q_{s}\right)$. If $f_{\omega}$ is infinite then we let $D\left(f_{\omega}\right)=\bigcup_{\tau<f_{\omega}} D(\tau)$.

Lemma 3.1. For all $s \leqslant \omega, D\left(f_{s}\right) \subseteq Q_{s}$. If $Q_{s}$ is finite then $Q_{s}=D\left(f_{s}\right)$ and $f_{s}$ is a leaf of $T_{s}$.

Proof. We already know that $a(\tau) \in Q_{s}$ if and only if $\tau \leqslant f_{s}$ if and only if $a(\tau) \in$ $D\left(f_{s}\right)$. Suppose that $F \subset Q_{s}$ is finite. For any finite $\tau \leqslant f_{s}, \bigcap_{m \in F} X_{m, s}$ is nonempty and intersects $X_{a(\tau), s}$, so there is some $\tau^{\prime} \geqslant \tau$ such that $F \subseteq D\left(\tau^{\prime}\right)$. Since $D(\tau) \subseteq D\left(\tau^{\prime}\right)$ this shows that for all $n \in D(\tau), X_{n, s}$ intersects $\bigcap_{m \in F} X_{m, s}$ for all finite $F \subseteq Q_{s}$; by maximality, $n \in Q_{s}$. So we have shown that $D(\tau) \subseteq D\left(f_{s}\right)$ for all finite $\tau \leqslant f_{s}$, so $D\left(f_{s}\right) \subseteq Q_{s}$.

Suppose that $Q_{s}$ is finite. Apply the reasoning above to $F=Q_{s}$ and $\tau=f_{s}$ and get some $\tau^{\prime} \geqslant f_{s}$ on $T_{s}$ such that $Q_{s} \subseteq D\left(\tau^{\prime}\right)$. But $D\left(\tau^{\prime}\right)$ is an index-set of a solution for $\mathcal{X}_{s}$; by maximality, $Q_{s}=D\left(\tau^{\prime}\right)$, and so by definition, $\tau^{\prime}=f_{s}$. If $f_{s}$ is not a leaf of $T_{s}$ then $D\left(f_{s}\right)$ is not maximal.

Where we use $\Delta_{2}^{0}$. The most expansive construction would carry out $(*)$ - adding $\tau^{\wedge} e$ to $T_{s+1}$ - whenever possible, for all appropriate strings $\tau \in T_{s}$ and all $e \leqslant s$ : the only necessary conditions are that $e \notin$ range $\tau$ (no need to force into $W_{e}$ again if we already have); that $\tau^{\wedge} e$ is not already on $T_{s}$; and that some proper extension of $\Gamma(\tau)$ is found in $W_{e, s}$. 
Suppose that $Q_{\omega}$ is an index-set of a maximal solution for $\mathcal{X}_{\omega}$. Any oracle which can enumerate $Q_{\omega}$ can compute the path $f_{\omega}$ and then compute $\Gamma\left(f_{\omega}\right)=\bigcup_{\tau \leqslant f_{\omega}} \Gamma(\tau)$; we assume for now that $f_{\omega}$ is infinite. If $e \in$ range $f_{\omega}$ then $\Gamma\left(f_{\omega}\right)$ meets $W_{e}$. On the other hand suppose that $W_{e}$ is dense around $\Gamma\left(f_{\omega}\right)$. If the construction is maximally expansive then for all $\tau \prec f_{\omega}$, if $e \notin$ range $\tau$ then $\tau^{\wedge} e \in T$. This implies that $X_{b(e)}$ intersects $\bigcap \mathcal{D}(\tau)$. This would imply that $b(e) \in Q_{\omega}$, provided that $Q_{\omega}=D\left(f_{\omega}\right)$. This is an important "niceness" property of $Q_{\omega}$. We already know that $a(\tau) \in Q_{\omega}$ if and only if $\tau<f_{\omega}$ and that $D\left(f_{\omega}\right) \subseteq Q_{\omega}$. So the question is whether there is some $b(e) \in Q_{\omega}$ such that $e \notin$ range $f_{\omega}$. If not then the construction succeeds: if $\Gamma\left(f_{\omega}\right)$ is in the closure of $W_{e}$ then $b(e) \in Q_{\omega}$ which implies that $e \in$ range $f_{\omega}$ which implies that $\Gamma\left(f_{\omega}\right)$ meets $W_{e}$.

This is the crux of the issue: if we could show that any maximal solution for the "maximally expansive" $\mathcal{X}$ is nice then we will have shown that every FIPdegree computes a 1-generic set. However there is no reason to assume that we get this niceness. This is where we use the assumption that the FIP-degree under consideration is $\Delta_{2}^{0}$.

Let $\mathbf{d}$ be a $\Delta_{2}^{0}$ FIP-degree. By Proposition 1.5 and the recursion theorem, during the construction we have access to a computable approximation of a d-computable, maximal FIP-solution $\mathcal{Q}_{\omega}$ for $\mathcal{X}=\mathcal{X}_{\omega}$ with index-set $Q_{\omega}$.

Claim 3.2. From $\mathcal{Q}_{\omega}$ we can obtain a sequence $\left\langle Q_{s}\right\rangle$ such that each $\mathcal{Q}_{s}$ is a maximal FIP-solution for $\mathcal{X}_{s}$, and such that for all $m \in Q, m \in Q_{s}$ for almost all $s$.

Proof. Let $\left\langle\overline{\mathcal{Q}}_{s}\right\rangle$ be a computable approximation of $\mathcal{Q}_{\omega}$ (recall that we can think of $\mathcal{Q}_{\omega}$ as an enumeration of $Q_{\omega}$ ). We can guarantee that for all $s$ there is some $t \geqslant s$ such that some initial segment of $\overline{\mathcal{Q}}_{t}$ is a maximal solution for $\mathcal{X}_{s}$. At stage $s$ before we do anything else we wait for such $t$ to appear. If no such $t$ ever appears then $\mathcal{X}_{\omega}=\mathcal{X}_{s}$. But then $\mathcal{Q}_{\omega}$ is an enumeration of a finite family and some finite initial segment of $\mathcal{Q}_{\omega}$ enumerates the entire family, which is a maximal solution for $\mathcal{X}_{s}$; this is a contradiction.

We thus let $\mathcal{Q}_{s}$ be such an initial segment (the first we find). It remains to show that $\lim _{s} \mathcal{Q}_{s}=\mathcal{Q}_{\omega}$, which implies that every $m \in Q_{\omega}$ lies in almost every $Q_{s}$. This clearly holds if the lengths of $\mathcal{Q}_{s}$ (as initial segments of the $\overline{\mathcal{Q}}_{t}$ ) tend to $\infty$. Otherwise there is a constant subsequence of the $\mathcal{Q}_{s}$, and this constant value is a finite maximal solution for infinitely many $\mathcal{X}_{s}$, and so is a maximal solution for $\mathcal{X}_{\omega}$. This solution is contained in $\mathcal{Q}_{\omega}$ and so equals $\mathcal{Q}_{\omega}$.

Now we only need to ensure that $\Gamma\left(Q_{\omega}\right)$ is 1-generic for this particular solution $Q_{\omega}$ which is gradually revealed to us during the construction; we do not need to perform the maximally expansive construction. If $b(e) \in Q_{\omega}$ then $b(e) \in Q_{s}$ for almost all $s$. As analysed above, if $b(e) \in Q_{s}$ then $e \in \operatorname{range} f_{s}$. Because of our restricted expansion, we will be able to show that if $e \in \operatorname{range} f_{s}$ for almost every $s$, then $e \in$ range $f_{\omega}$ as required.

Ensuring the path is infinite. There is one last issue which we have not yet discussed, which is ensuring that $f_{\omega}$ is infinite. If we are not careful then the finite paths $f_{s}$ could get arbitrarily long, extending a finite $f_{\omega}$, but $Q_{\omega}$ may be infinite, containing infinitely many $b(e)$ 's but only $a(\tau)$ for $\tau \leqslant f_{\omega}$. For any finite set $F$ of these $b(e)$ 's there is an extension $\tau$ of $f_{\omega}$ such that $F \subset D(\tau)$, but the approximation does not settle on any one of these strings $\tau$. 
We counter that problem by ensuring that $f_{\omega}$ has only finitely many immediate extensions on $T$. This we do by imposing restraint on which $\tau^{\wedge} e$ can be enumerated into $T$; if $\tau^{\wedge} d$ is already on $T$ and $d<e$ then we postpone trying to force into $W_{e}$ and only enumerate $\sigma^{\wedge} e$ for longer strings $\sigma$. This introduces some finite injury but is of course easily handled. The effect would be that one of the immediate successors $\tau$ of $f_{\omega}$ must occur in infinitely many $f_{s}$. Maximality of $Q_{\omega}$ will show that $a(\tau) \in Q_{\omega}$.

3.2.2. Construction. At stage $s$, for any $e \leqslant s$ and $\tau \leqslant f_{s}$ such that:

(i) $e \notin$ range $\tau$;

(ii) there is no $d \leqslant e$ with $\tau^{\wedge} d \in T_{s}$; and

(iii) there is some string $\rho$ in $W_{e, s}$ properly extending $\Gamma(\tau)$,

we enumerate $\tau^{\wedge} e$ into $T_{s+1}$ and define $\Gamma\left(\tau^{\wedge} e\right)=\rho$. As discussed above this determines new enumerations into $\mathcal{X}$.

3.2.3. Verification. Observe that $T$ is finitely branching: if $\tau^{\wedge} e$ is the first extension of $\tau$ enumerated into $T$ then $\tau$ has at most $e+1$ many immediate extensions on $T$.

Claim 3.3. $f_{\omega}$ is infinite.

Proof. We first show that $Q_{\omega}$ is infinite. Otherwise $f_{\omega}$ is a leaf of $T$ (Lemma 3.1). In this case for almost all $s, f_{s}=f_{\omega}$. There is some $e \notin$ range $f_{\omega}$ such that $W_{e}$ contains a proper extension of $\Gamma\left(f_{\omega}\right)$. If this appears by stage $s$ and $f_{s}=f_{\omega}$ then at stage $s$ we enumerate $f_{\omega}{ }^{\wedge} e$ into $T$, a contradiction.

Now suppose for a contradiction that $f_{\omega}$ is finite despite $Q_{\omega}$ being infinite. For almost all $s, f_{s}$ extends $f_{\omega}$. Let $F \subset Q_{\omega}$ be finite. For almost all $s, F \subseteq Q_{s}=D\left(f_{s}\right)$. Since for most such $F$ we will have $F \nsubseteq D\left(f_{\omega}\right)$, for almost all $s, f_{s}$ is a proper extension of $f_{\omega}$, and so contains one of the immediate extensions of $f_{\omega}$ on $T$. Since there are only finitely many of these, there is an immediate extension $\tau$ of $f_{\omega}$ such that $\tau \leqslant f_{s}$ for infinitely many $s$. Again let $F \subset Q_{\omega}$ be finite. Suppose that $s$ is large and that $\tau \leqslant f_{s}$. Then $F \subseteq D\left(f_{s}\right)$ and so $\bigcap \mathcal{F}$ intersects $X_{a(\tau)}$. By maximality of $Q_{\omega}, a(\tau) \in Q_{\omega}$, contradicting the definition of $f_{\omega}$.

We can now show that $Q_{\omega}$ is nice. This directly relies on the fact that we only try to attack what we believe to be $Q$ at any stage and do not perform an expansive construction.

Claim 3.4. $Q_{\omega}=D\left(f_{\omega}\right)$.

Proof. We show that there are infinitely many stages $s$ at which $f_{s}<f_{\omega}$. This implies the claim since every element of $Q$ is an element of $Q_{s}$ for almost all $s$; if $f_{s}<f_{\omega}$ then $Q_{s}=D\left(f_{s}\right) \subset D\left(f_{\omega}\right)$.

Let $t_{0}$ be any stage. Let $\sigma=T_{t_{0}} \cap f_{\omega}$ be the longest initial segment of $f_{\omega}$ on $T_{t_{0}}$. Let $\tau=\sigma^{\wedge} f_{\omega}(|\sigma|)$ be the initial segment of $f_{\omega}$ which extends $\sigma$ by one element. Let $t_{1}>t_{0}$ be the least stage after $t_{0}$ such that $\tau \in T_{t_{1}}$; so $\tau$ is a leaf of $T_{t_{1}}$. If $\tau=f_{t_{1}}$ then we are done. Otherwise let $s>t_{1}$ be the least stage after $t_{1}$ at which $\tau \leqslant f_{s}$. Since $\tau$ is not an initial segment of $f_{r}$ for any stage $r \in\left[t_{1}, s\right)$, no extensions of $\tau$ are enumerated into $T$ at any such stage $r$. Hence $\tau$ is a leaf of $T_{s}$, whence $\tau=f_{s}$, and $s$ is a stage as required.

The rest of the proof mostly proceeds as described above. Since $\Gamma$ is strictly monotone and $f_{\omega}$ is infinite, $G=\Gamma\left(f_{\omega}\right)$ is an element of $2^{\omega}$. As we mentioned 
above, $\mathbf{d}$ can enumerate $Q$ and so compute $f_{\omega}$ and so compute $G$. We want to show that $G$ meets or avoids $W_{e}$. Since $Q$ is nice, it suffices to show that if $W_{e}$ is dense around $G$ then $b(e) \in Q$; this would imply that $e \in \operatorname{range} f_{\omega}$. To deal with the finite injury we need to show this inductively.

Claim 3.5. For every $e$ there are only finitely many $\tau<f_{\omega}$ such that $\tau^{\wedge} e \in T$; and $G$ meets or avoids $W_{e}$.

Proof. By induction on $e$. Suppose this is known for all $e^{\prime}<e$. If $G$ avoids $W_{e}$ then for some $\tau<f_{\omega}, \Gamma(\tau)$ has no extension in $W_{e}$. Then for all $\sigma \geqslant \tau$ on $T, \sigma^{\wedge} e \notin T$. If $e \in$ range $f_{\omega}$ then $G$ meets $W_{e}$; if $\tau<f_{\omega}$ and $e \in \operatorname{range} \tau$ then again for all $\sigma \geqslant \tau$ on $T, \sigma^{\wedge} e \notin T$.

We show that one of these cases must happen. If not then for every $\tau<f_{\omega}$, $\Gamma(\tau)$ has a proper extension in $W_{e}$. By induction, for all but finitely many $\tau<f_{\omega}$, $\tau^{\wedge} d \notin T$ for all $d<e$. For each such $\tau$, we will eventually see a late stage $s$ such that $\tau \leqslant f_{s}$ and we also see an extension of $\Gamma(\tau)$ in $W_{e}$; at such a stage, since $e \notin$ range $\tau$, we enumerate $\tau^{\wedge} e$ into $T$. This shows that $X_{b(e)}$ intersects $D(\tau)$ for all $\tau<f_{\omega}$. Since $Q_{\omega}=D\left(f_{\omega}\right)$, maximality of $Q_{\omega}$ shows that $b(e) \in Q_{\omega}$. Again since $Q_{\omega}=D\left(f_{\omega}\right)$ this shows that $e \in$ range $f_{\omega}$ after all, a contradiction.

This concludes the verification, and so the proof of the fact that every $\Delta_{2}^{0}$ FIPdegree computes a 1-generic set.

3.3. Genericity and $\Delta_{2}^{0}$ 2IP degrees. We now show how to modify the preceding argument to prove Theorem 1.4. We start of course by observing what goes wrong if we just take a 2IP-solution to the family $\mathcal{X}$ enumerated above and hope to run the same argument.

The first place where we run into a serious difficulty is when trying to prove Lemma 3.1. It is possible that, for example, $b\left(e_{0}\right), b\left(e_{1}\right), b\left(e_{2}\right)$ are all elements of $Q_{s}$, but $\bigcap_{i=0,1,2} X_{b\left(e_{i}\right)}$ is empty. It is possible that $T_{s}$ contains the strings $\tau^{\wedge}\left\langle e_{0}, e_{1}\right\rangle$, $\tau^{\wedge}\left\langle e_{0}, e_{2}\right\rangle$ and $\tau^{\wedge}\left\langle e_{1}, e_{2}\right\rangle$ but not any string containing all of $e_{0}, e_{1}$ and $e_{2}$. We could then have $Q_{s}$ properly containing $D(\tau)$ (where $\tau=f_{s}$ ) and so would not be "nice".

The solution for this problem is in a sense to force such $Q_{s}$ to be nice by "giving it more opportunity" to be nice. Namely in that situation we add a new 1-bit extension $\tau^{\prime}$ to $\tau$ coding this possibility and intersect $X_{a\left(\tau^{\prime}\right)}$ with $\bigcap_{i=0,1,2} X_{b\left(e_{i}\right)}$. A solution containing all $X_{b\left(e_{i}\right)}$ (and $X_{a(\tau)}$ ) will not be maximal unless it also contains $X_{a\left(\tau^{\prime}\right)}$. We would then have $f_{s}=\tau^{\prime}$ and $Q_{s}$ will be nice again.

Of course we still need to make progress on some requirement, even if the option $\tau^{\prime}$ is taken. Since each $e_{i}$ appears in some extension of $\tau$ we already know that $\Gamma(\tau)$ has extensions in each $W_{e_{i}}$. We choose one $e_{i}$, say $e_{0}$, and let $\Gamma\left(\tau^{\prime}\right)$ be an extension of $\Gamma(\tau)$ in $W_{e_{0}}$; we cannot hope to deal with more than one $W_{e_{i}}$ since the various extensions could be incomparable.

This creates a new problem: the sets $X_{b\left(e_{1}\right)}$ and $X_{b\left(e_{2}\right)}$ are now useless for forcing the solution to meet $W_{e_{1}}$ or $W_{e_{2}}$ when the solution contains $\tau^{\prime}$. We have already thrown them into the intersection but did not use that to meet the corresponding requirements. As a result, when working above $\tau^{\prime}$ we need to discard the indices $b\left(e_{1}\right)$ and $b\left(e_{2}\right)$ and choose fresh indices $b^{\prime}\left(e_{1}\right)$ and $b^{\prime}\left(e_{2}\right)$ to take the role of $b\left(e_{1}\right)$ and $b\left(e_{2}\right)$ above $\tau^{\prime}$. If we don't do this stupidly, i.e. if we choose $e_{0}$ smaller than $e_{1}$ and $e_{2}$, then this only introduces finite injury over the previous construction and does not trouble us too badly. 
3.3.1. The set-up and construction. As in the FIP-case we enumerate a c.e. tree $T$, define a map $\Gamma: T \rightarrow 2^{<\omega}$, and enumerate a family $\mathcal{X}$. The strings on the tree will not be strings of natural numbers but of pairs $(e, F)$ consisting of a natural number $e$ and a finite set $F$ of natural numbers such that either $F=\varnothing$ or $e<\min F$. If $\tau$ is a nonempty sequence of such pairs we let $(e(\tau), F(\tau))$ be the pair $(e, F)$ which appears at the end of $\tau$ (the "last bit" of $\tau$ ). We will ensure that for all nonempty $\tau \in T$, $\Gamma(\tau) \in W_{e(\tau)}$. We will ensure that the sequence $\langle e(\sigma)\rangle_{\sigma \leqslant \tau}$ is injective.

The elements of $\mathcal{X}$ will be indexed by $a(\tau)$ for $\tau \in T$ and numbers $b(d, \tau)$ for nonempty $\tau \in T$. However the numbers $b(d, \tau)$ and $b\left(d, \tau^{\prime}\right)$ are not always distinct. $b(d, \tau)$ is the version of $b(d)$ which $\tau$ gets to work with. The guiding rule, as discussed above, is: extensions of $\tau$ need to get new versions of $b(d)$ for all $d>e(\tau)$. Thus we define $b(d, \tau)$ by recursion on the length of $\tau$. For any $\tau \in T$ and $d<\omega$ we will define $b\left(d, \tau^{+}\right)$, the indices provided by $\tau$ to its immediate successors; if $\sigma$ is an immediate successor of $\tau$ then $b(d, \sigma)=b\left(d, \tau^{+}\right)$. The numbers $b(d,\langle\rangle)$ are not defined. We start by choosing a fresh $b\left(d,\langle\rangle^{+}\right)$for every $d$. If $\tau$ is nonempty and $b(d, \tau)$ are already defined then for $d \leqslant e(\tau)$ we let $b\left(d, \tau^{+}\right)=b(d, \tau)$ but for $d>e(\tau)$ we choose a new value for $b\left(d, \tau^{+}\right)$.

We can then define for $\tau \in T$

$$
D(\tau)=\{a(\sigma): \sigma \leqslant \tau\} \cup\{b(e(\sigma), \sigma): \sigma \leqslant \tau\} \cup\{b(d, \sigma): \sigma \leqslant \tau \& d \in F(\sigma)\} .
$$

Since $e(\langle\rangle)$ and $F(\langle\rangle)$ are not defined, $D(\langle\rangle)=\{a(\langle\rangle)\}$.

The family $\mathcal{X}$ is determined by $T$ : when a string $\tau$ is added to $T$ we enumerate a number into $\bigcap \mathcal{D}(\tau)$. We will ensure that for $s<\omega, T_{s}$ is finite, and so only finitely many sets in $\mathcal{X}_{s}$ are nonempty.

As above we are given a $\Delta_{2}^{0}$ 2IP-degree $\mathbf{d}$ and a $\mathbf{d}$-c.e. index-set $Q_{\omega}$ for a maximal 2IP-solution for $\mathcal{X}=\mathcal{X}_{\omega}$. The proof of Claim 3.2 holds for the current construction as well; we obtain a sequence $\left\langle Q_{s}\right\rangle$ such that each $Q_{s}$ is an index-set for a maximal 2IP-solution for $\mathcal{X}_{s}$ and for all $m$, if $m \in Q_{\omega}$ then $m \in Q_{s}$ for almost all $s$.

Let $\tau, \tau^{\prime} \in T$. As in the previous construction we observe that if $\tau$ and $\tau^{\prime}$ are incomparable then $X_{a(\tau)}$ and $X_{a\left(\tau^{\prime}\right)}$ are disjoint, but that if $\tau \leqslant \tau^{\prime}$ then $D(\tau) \subseteq$ $D\left(\tau^{\prime}\right)$. Also $X_{a(\langle)}$ is nonempty and intersects every nonempty set in $\mathcal{X}_{s}$. Let $s \leqslant \omega$. Then $a(\langle\rangle) \in Q_{s}$. Suppose that $a\left(\tau^{\prime}\right) \in Q_{s}$ and that $\tau \leqslant \tau^{\prime}$. Let $m \in Q_{s}$. Then $X_{m} \cap X_{a\left(\tau^{\prime}\right)}$ is nonempty, which means that $m \in D\left(\tau^{\prime \prime}\right)$ for some $\tau^{\prime \prime} \geqslant \tau^{\prime}$ in $T_{s}$. Then $X_{m} \cap X_{a(\tau)}$ is nonempty since $a(\tau) \in D\left(\tau^{\prime \prime}\right)$. Thus $a(\tau) \in Q_{s}$. It follows that $\left\{\tau: a(\tau) \in Q_{s}\right\}$ is a nonempty path of $T_{s}$, possibly finite, which we again name $f_{s}$. We will need to work a little more to prove an analogue of Lemma 3.1 and so we postpone this to the verification.

We now state the construction. We start with $T_{0}$ consisting of the empty sequence, mapped by $\Gamma$ to the empty sequence. Stage $s \geqslant 0$ consists of two parts.

(a) For all $\tau \leqslant f_{s}$ and $e \leqslant s$ such that:

(i) For all $\sigma \leqslant \tau, e \neq e(\sigma)$;

(ii) For all immediate extensions $\sigma$ of $\tau$ on $T, e(\sigma)>e$; and

(iii) There is a proper extension $\rho$ of $\Gamma(\tau)$ in $W_{e, s}$ we enumerate $\tau^{\wedge}(e, \varnothing)$ into $T$ and map it to $\rho$.

(b) For any finite set $F$ consisting of at least two numbers and any $\tau \in T_{s}$ such that for all $d \in F$ there is some $\nu \geqslant \tau$ such that:

(i) $\nu^{\wedge}(d, \varnothing)$ is currently on $T$ (either in $T_{s}$ or added during step (a)); and 
(ii) $b\left(d, \nu^{+}\right)=b\left(d, \tau^{+}\right)$

we let $d=\min F$ and enumerate $\tau^{\wedge}(d, F-\{d\})$ into $T$ and map it to $\Gamma\left(\nu^{\wedge}(d, \varnothing)\right)$ where $\nu$ witnesses that $d \in F$.

\section{Verification.}

Lemma 3.6. Let $\tau \in T$.

(1) The sequence $\langle e(\sigma)\rangle_{\sigma \leqslant \tau}$ is injective.

(2) $\Gamma(\tau) \in W_{e(\tau)}$.

(3) For all $\sigma<\tau, \Gamma(\sigma)<\Gamma(\tau)$.

Proof. By induction on the stages we show this for all $\tau \in T_{s}$. All three are clear for strings $\tau^{\wedge}(d, \varnothing)$ is enumerated into $T$ at step (a) of stage $s$. Suppose that $\tau^{\wedge}(d, F)$ is added to $T$ at step (b) of stage $s$. Then $\nu^{\wedge}(d, \varnothing)$ is already on $T$, where $\nu \geqslant \tau$. By induction $e(\sigma) \neq d$ for all $\sigma \leqslant \nu$ and hence all $\sigma \leqslant \tau$. (2) follows from the fact that $\Gamma\left(\tau^{\wedge}(d, F)\right)=\Gamma\left(\nu^{\wedge}(d, \varnothing)\right)$ which is in $W_{d}$. (3) follows by induction since $\Gamma(\tau) \leqslant \Gamma(\nu)$ and $\Gamma(\nu)$ is properly extended by $\Gamma\left(\nu^{\wedge}(d, \varnothing)\right)$.

For the following claim, let $d \geqslant 0$ and let $\tau \in T$ be nonempty. Consider the shortest initial segment $\mu$ of $\tau$ such that $b(d, \tau)=b\left(d, \mu^{+}\right)$. This is the string which introduced the index $b(d, \tau)$ to its successors. If this string $\mu$ is nonempty then it is the longest proper initial segment $\mu$ of $\tau$ such that $e(\mu)<d$; if it is empty then $e(\mu) \geqslant d$ for all nonempty proper initial segments $\mu$ of $\tau$. If $\sigma$ is any string such that $b\left(d^{\prime}, \sigma\right)=b(d, \tau)$ then $d^{\prime}=d$ and $\sigma$ also properly extends $\mu$.

Claim 3.7. Let $s \leqslant \omega$. Let $d<\omega$ and let $\tau \in T_{s}$ be nonempty; let $m=b(d, \tau)$. Let $\mu$ be the shortest initial segment of $\tau$ such that $m=b\left(d, \mu^{+}\right)$. Let $n<\omega$. If $X_{n, s}$ intersects $X_{m, s}$ then it also intersects $X_{a(\mu), s}$ (and in fact intersects $X_{a(\sigma), s}$ for some immediate successor $\sigma$ of $\mu$ on $T_{s}$.)

Proof. Since $X_{n, s}$ and $X_{m, s}$ intersect there is some $\sigma_{1} \in T_{s}$ such that $n, m \in D\left(\sigma_{1}\right)$. Then $m=b\left(d, \sigma_{0}\right)$ for some $\sigma_{0} \leqslant \sigma_{1}$. $\sigma_{0}$ properly extends $\mu$ and $X_{n, s}$ intersects $X_{a(\sigma), s}$ for all $\sigma \leqslant \sigma_{1}$.

Let $s \leqslant \omega$ and let $\tau \leqslant f_{s}$ be finite. Let $m \in Q_{s}$ and let $n \in D(\tau)$. Then $X_{m, s}$ intersects $X_{a(\tau), s}$. Hence $m \in D\left(\tau^{\prime}\right)$ for some $\tau^{\prime} \geqslant \tau$. It follows that $X_{m, s}$ intersects $X_{n, s}$. We conclude that $D\left(f_{s}\right) \subseteq Q_{s}$.

Claim 3.8. Let $s \leqslant \omega$ be such that $f_{s}$ is finite. Every $m \in Q_{s}-D\left(f_{s}\right)$ is of the form $b\left(d, f_{s}^{+}\right)$for some $d$, and there is some $\nu \geqslant f_{s}$ such that $m=b\left(d, \nu^{+}\right)$and $\nu^{\wedge}(d, \varnothing) \in T_{s}$.

Proof. Let $m \in Q_{s}-D\left(f_{s}\right)$. Since $X_{m, s}$ intersects $X_{a\left(f_{s}\right), s}$ we know that $m \in D\left(\sigma_{1}\right)$ for some $\sigma_{1} \geqslant f_{s} . m$ cannot equal $a(\sigma)$ for any $\sigma$ and so there is some $\sigma_{0}$ and some pair $(e, F)$ such that $\sigma_{0}{ }^{\wedge}(e, F) \leqslant \sigma_{1}, m=b\left(d, \sigma_{0}^{+}\right)$and $d \in\{e\} \cup F$. Since $m \notin D\left(f_{s}\right)$, $\sigma_{0} \geqslant f_{s}$.

By the instructions, there is some $\nu \geqslant \sigma_{0}$ such that $m=b\left(d, \nu^{+}\right)$and $\nu^{\wedge}(d, \varnothing) \in$ $T_{s}$ : If $F=\varnothing$ then we of course take $\nu=\sigma_{0}$. Otherwise $\sigma_{0}{ }^{\wedge}(e, F)$ is added at step (b) of some stage $t<s$ and then we take $\nu$ to be a witness for $d \in\{e\} \cup F$.

Let $\mu$ be the shortest initial segment of $\sigma_{0}$ such that $m=b\left(d, \mu^{+}\right)$. Since $Q_{s}$ is a solution, Claim 3.7 says that for all $n \in Q_{s}, X_{n, s}$ intersects $X_{a(\mu), s}$. By maximality of $Q_{s}, a(\mu) \in Q_{s}$. Hence $\mu \leqslant f_{s}$. Since $f_{s} \leqslant \sigma_{0}$ and $m=b\left(d, \sigma_{0}^{+}\right)$, we conclude that $m=b\left(d, f_{s}^{+}\right)$. 
Lemma 3.9. Let $s \leqslant \omega$. If $Q_{s}$ is finite then $Q_{s}=D\left(f_{s}\right)$ and $f_{s}$ is a leaf of $T_{s}$.

Proof. Let $F$ be the set of numbers $d$ such that $b\left(d, f_{s}^{+}\right) \in Q_{s}-D\left(f_{s}\right)$, which we assume is nonempty. If $F=\{d\}$ is a singleton, find some $\nu \geqslant f_{s}$ such that $b\left(d, f_{s}^{+}\right)=b\left(d, \nu^{+}\right)$and $\tau=\nu^{\wedge}(d, \varnothing) \in T_{s}$ (Claim 3.8). Then $Q_{s} \subseteq D(\tau)$; by maximality, $Q_{s}=D(\tau)$, a contradiction.

Suppose that $F$ is not a singleton. Let $d^{*}=\min F$. Since for every $d \in F$ there is some $\nu \geqslant f_{s}$ such that $b\left(d, f_{s}^{+}\right)=b\left(d, \nu^{+}\right)$and $\nu^{\wedge}(d, \varnothing) \in T_{s}$, by step (b) of stage $s-1$ we will have enumerated $\tau=f_{s}{ }^{\wedge}\left(d^{*}, F-\left\{d^{*}\right\}\right)$ into $T_{s}$. This requires that $f_{s} \in T_{s-1}$, but this holds because $f_{s}<\nu^{\wedge}(d, \varnothing) \in T_{s}$ shows that $f_{s}$ is not a leaf of $T_{s}$, and all elements of $T_{s}-T_{s-1}$ are leaves by construction. But then again $Q_{s} \subseteq D(\tau)$ which is impossible.

Claim 3.10. Let $\tau \in T$. There are only finitely many numbers $d$ such that $b\left(d, \tau^{+}\right)$ is an element of $D\left(\tau^{\prime}\right)$ for any proper extension $\tau^{\prime}$ of $\tau$.

Proof. We assume that $\tau$ is not a leaf of $T$. The first proper extension of $\tau$ ever enumerated into $T$ is of the form $\tau^{\wedge}(c, \varnothing)$ for some $c<\omega$. By induction on the stages we show that if $b\left(d, \tau^{+}\right) \in D\left(\tau^{\prime}\right)$ and $\tau^{\prime}$ properly extends $\tau$ then $d \leqslant c$.

As in the previous construction, if $\tau^{\wedge}(d, \varnothing) \in T$ then $d \leqslant c$.

If $\tau^{\wedge}(d, F)$ is added to $T$ where $F \neq \varnothing$ then for all $e \in\{d\} \cup F, b\left(e, \tau^{+}\right)$is in $D\left(\tau^{\prime}\right)$ for some proper extension $\tau^{\prime}$ of $\tau$ which is already on $T$. By induction, every element of $\{d\} \cup F$ is bounded by $c$.

Now consider extensions of $\tau$ which are not immediate extensions. Suppose that $\sigma$ is a proper extension of $\tau$ and that an immediate extension of $\sigma$ is added to $T$. Say $\tau^{\wedge}(e, G) \leqslant \sigma$. By induction $e \leqslant c$. If $b\left(d, \sigma^{+}\right)=b\left(d, \tau^{+}\right)$then $d \leqslant e$.

As a consequence, $T$ is finitely branching. We know that every node $\tau \in T$ has only finitely many immediate extensions of the form $\tau^{\wedge}(d, \varnothing)$. If $\tau^{\wedge}(d, F) \in T$ where $F$ is nonempty then for all $e \in\{d\} \cup F, b\left(e, \tau^{+}\right)$belongs to some $D\left(\tau^{\prime}\right)$ for some proper extension $\tau^{\prime}$ of $\tau$. So there can only be finitely many such sets $F$.

Lemma 3.11. $f_{\omega}$ is infinite.

Proof. Suppose, for a contradiction, that $f_{\omega}$ is finite. Claims 3.8 and 3.10 together imply that $Q_{\omega}-D\left(f_{\omega}\right)$ is finite, and so that $Q_{\omega}$ is finite. By Lemma $3.9, f_{\omega}$ is a leaf of $T$.

We then argue as in the previous construction (Claim 3.3). There is some $e$ such that $e \neq e(\sigma)$ for all $\sigma \leqslant f_{\omega}$ and $W_{e}$ contains a proper extension of $\Gamma\left(f_{\omega}\right)$. This shows that at some stage a proper extension of $f_{\omega}$ is enumerated into $T$, a contradiction.

It follows that $G=\Gamma\left(f_{\omega}\right) \in 2^{\omega}$. As in the previous construction, $G$ is $\mathbf{d}$ computable. The rest of the verification follows as in the previous construction. This completes the proof of Theorem 1.4.

\section{VARIANTS}

4.1. Finite variants. A natural question to ask is what happens if we require every set in the given family to be finite. In some cases the answer is not interesting. For example:

Proposition 4.1. Suppose that $\mathcal{A}$ is a computable family consisting of finite sets. Then every maximal FIP-solution for $\mathcal{A}$ is computable. 
Proof. Let $\mathcal{Q}$ be a maximal FIP-solution for $\mathcal{A}$. Then in fact the intersection $\bigcap \mathcal{Q}$ of all the sets in $\mathcal{Q}$ is nonempty (a finite discrete space is compact). Let $F=\bigcap \mathcal{Q}$. Then the collection of all sets in $\mathcal{A}$ which contain $F$ is a computable FIP-solution. By maximality, it equals $\mathcal{Q}$.

Here the behaviour of FIP and 2IP diverges.

Proposition 4.2. Suppose that $\mathcal{A}$ is a computable family consisting of finite sets. Then $\mathcal{A}$ has a computable maximal 2IP-solution. However, there is a computable family consisting of finite sets which has continuum many maximal 2IP-solutions, and hence noncomputable ones.

Proof. For the first part, let $\mathcal{A}$ be a computable family consisting of finite sets (we have a computable index for each set but not a canonical (strong) index as a finite set). We enumerate an index-set $Q$ for a maximal 2IP-solution, essentially by repeating a greedy algorithm. By stage $s$ we have enumerated $Q_{s}$. If there is one, we add to $Q$ the least $n \leqslant s$ which is not already in $Q_{s}$ such that $A_{n} \cap A_{m} \cap[0, s]$ is nonempty for all $m \in Q_{s}$. To see that this gives a maximal solution consider any $n \notin Q$. Let $t>\max A_{n}$ be sufficiently late so that $Q_{t} \uparrow_{n}=Q \uparrow_{n}$. Then there is some $m \in Q_{t}$ such that $A_{n} \cap A_{m} \cap[0, t]$ is empty. But this means that $A_{n} \cap A_{m}$ is empty.

We now construct a computable family $\mathcal{X}$ which consists of finite sets but has continuum many maximal 2IP-solutions. We index the sets in $\mathcal{X}$ by finite binary strings $\sigma \in 2^{<\omega}$ rather than by natural numbers. First let $\left\{a_{\sigma, i}: \sigma \in 2^{<\omega}, i<2\right\}$ be a partition of $\omega$. Let $X_{\langle>}=\left\{a_{\diamond>, 0}, a_{\diamond>, 1}\right\}$. Let $\sigma \in 2^{<\omega}$ be nonempty; let $j$ be the last bit of $\sigma$. We let $X_{\sigma}=\left\{a_{\sigma, 0}, a_{\sigma, 1}\right\} \cup\left\{a_{\tau, j}: \tau \npreceq \sigma\right\}$.

The important properties of these sets are: (1) if $\sigma \leqslant \tau$ then $X_{\sigma}$ and $X_{\tau}$ intersect; and (2) for all $\sigma, X_{\sigma^{\wedge} 0}$ and $X_{\sigma^{\wedge} 1}$ are disjoint. For every $f \in 2^{\omega},\left\{X_{\left.f\right|_{n}}: n<\omega\right\}$ is a 2IP-solution for $\mathcal{X}$, so is contained in some maximal 2IP-solution $\mathcal{Q}_{f}$. This will often be a larger solution; for example, each $X_{\sigma^{\wedge} 0}$ intersects every $X_{0^{n}}$. Nonetheless the maximal solutions $\mathcal{Q}_{f}$ are distinct; if $f, g \in 2^{\omega}$ are distinct and $\sigma=f \cap g$ then one of $\mathcal{Q}_{f}$ and $\mathcal{Q}_{g}$ contains $X_{\sigma^{\wedge} 0}$ and the other contains $X_{\sigma^{\wedge} 1}$, and no solution can contain both.

4.1.1. C.e. families. As mentioned early in the paper, for general intersection problems, in terms of computation power there is no difference between computable families and c.e. families. This breaks down if we insist that the families consist only of finite sets.

The proof of Proposition 4.1 holds for c.e. families as well. However 2IP behaves differently.

Proposition 4.3. There is a c.e. family $\mathcal{X}$ consisting of finite sets such that every degree which can enumerate a maximal 2IP-solution for $\mathcal{X}$ is hyperimmune.

Proof. We modify the proof given in Section 3.1. To make the sets in the family $\mathcal{X}$ finite we consider the priority between them. Again the sets have indices $b(e)$ and $a(e, k)$. Again each nonempty set contains an individual identifying marker. Recall that the required intersection pattern is:

(1) $X_{b(e)}$ is nonempty if and only if $\varphi_{e}(e) \downarrow$;

(2) $X_{b(e)}$ and $X_{a(e, k)}$ intersect if and only if both $\varphi_{e}(e) \downarrow$ and $\varphi_{e}(k) \downarrow$; 
(3) these are the only restrictions on intersections; in particular, every set $X_{a(e, k)}$ is nonempty.

We show how to achieve this pattern while keeping all sets finite. Apart from the individual markers we reserve numbers $r_{e}$ for all $e<\omega$ and pairs of numbers $p_{n}$ and $q_{n}$ for all $n<\omega$. We then act as follows:

(a) To intersect $X_{b(e)}$ with $X_{a(e, k)}$, we enumerate $r_{e}$ into both.

(b) If $n<m$ and we want to intersect $X_{n}$ and $X_{m}$ because of condition (3) then:

(i) if $X_{m}$ is a $X_{b(e)}$ then we enumerate $p_{n}$ into $X_{n} \cap X_{m}$;

(ii) if $X_{m}$ is a $X_{a(e, k)}$ then we enumerate $q_{n}$ into $X_{n} \cap X_{m}$.

The point is that we do not accidentally intersect $X_{b(e)}$ with $X_{a(e, k)}$ by acting for condition (3).

4.2. Bounded variants. An even stronger condition than all sets being finite is having a finite bound on the size of all sets. Here even coding into c.e. families fails.

Proposition 4.4. Let $N, n<\omega$. Let $\mathcal{A}$ be a c.e. family of sets, all of which have fewer than $N$ elements. Then every maximal $n$ IP-solution for $\mathcal{A}$ is computable.

Proof. Let $\mathcal{Q}$ be a maximal $n$ IP-solution for $\mathcal{A}$. We define a finite tree $T$, labelled by finite sets of numbers. We define it inductively, starting with $T_{0}$ consisting of a root labelled by the empty set. At step $s$ we have a finite tree $T_{s}$; we will either stop the process or add finitely many children to one of the leaves of $T_{s}$ to obtain $T_{s+1}$. We ensure that the following properties holds for every tree $T_{s}$ :

(1) Sets at level $k$ of $T_{s}$ have size $k$.

(2) For every set $X$ on $T_{s}$ there is some set $D \in \mathcal{Q}$ such that $X \subseteq D$.

(3) If $X$ is a set on $T_{s}$ which is not a leaf of $T_{s}, D \in \mathcal{Q}$ and $X \subseteq D$, then there is some child $Y$ of $X$ on $T_{s}$ such that $Y \subseteq D$.

Since we start by placing the empty set at the root, inductively, (3) implies that every set $D \in \mathcal{Q}$ contains some leaf of $T_{s}$. We stop the process when we have built a tree $T=T_{s}$ satisfying:

(4) Any $n$ leaves of $T$ have nonempty intersection.

If this is successful then the collection of sets in $\mathcal{A}$ which contain at least one leaf of $T$ is a computable $n$ IP-solution containing $\mathcal{Q}$; by maximality, it equals $\mathcal{Q}$.

As mentioned we start by placing the empty set at the root. Properties (1)-(3) are all trivially satisfied.

At step $s$, suppose that we have $T_{s}$, satisfying (1)-(3) but not (4). We show that there is a way to take a leaf $X$ of the current tree and add finitely many children $X \cup\{z\}$ (where $z \notin X$ ) so that (1)-(3) hold in the new tree $T_{s+1}$ as well. Let $T=\bigcup_{s} T_{s}$. The tree $T$ is finitely branching. Every set on $T$ is contained in some set from $\mathcal{Q}$ and so has fewer than $N$ many elements. By (1), $T$ has at most $N$ many levels, so $T$ is finite. Since each $T_{s+1}$ is strictly bigger than $T_{s}$ there is some $t$ such that $T=T_{t}$. This implies that $T_{t}$ satisfies (4), so the construction succeeds.

So consider $T_{s}$ failing (4). Let $X_{1}, X_{2}, \ldots, X_{n}$ be leaves of $T_{s}$ witnessing this failure (for example if $s=0$ then $X_{i}=\varnothing$ for all $i \leqslant n$ ). For every $\ell \in\{0,1,2, \ldots, n\}$ consider all intersections

$$
X_{1} \cap X_{2} \cap \cdots \cap X_{\ell} \cap D_{\ell+1} \cap \cdots \cap D_{n}
$$


where the sets $D_{\ell+1}, \ldots, D_{n}$ are chosen from $\mathcal{Q}$. For $\ell=0$ all the sets are chosen from $\mathcal{Q}$ and so we get nonempty intersections. For $\ell=n$ we simply get $X_{1} \cap \cdots X_{n}$ which is empty. So we let $\ell$ be the least such that some choice of $D_{\ell+1}, \ldots, D_{n}$ results in an empty intersection; so $0<\ell \leqslant n$ (if $s=0$ then $\ell=1$ ). Let $D_{\ell+1}, \ldots, D_{n}$ be such a choice.

Now let $X=X_{\ell}$. By choice of $\ell$, the set

$$
Z=X_{1} \cap X_{2} \cap \cdots X_{\ell-1} \cap D_{\ell+1} \cap \cdots \cap D_{n}
$$

is disjoint from $X$. We let

$$
Z^{\prime}=\{z \in Z \text { : there is some } D \in \mathcal{Q} \text { such that } X \cup\{z\} \subseteq D\} \text {. }
$$

Suppose that $D \in \mathcal{Q}$ and $X \subseteq D$. By choosing $D_{\ell}=D$, the minimality of $\ell$ shows that $D \cap Z$ is nonempty. Since there is such a set $D-(2)$ holds for $T_{s}$ - we see that $Z^{\prime}$ is nonempty. Certainly $Z$ is finite, as it is contained in some $X_{i}$ or some $D_{i}$. We then define $T_{s+1}$ by letting the children of $X$ be $X \cup\{z\}$ for $z \in Z^{\prime}$. Then:

- $T_{s+1} \neq T_{s}$, as $Z^{\prime}$ is nonempty.

- $T_{s+1}$ is finitely branching, since $Z^{\prime}$ is finite.

- (1) holds for $T_{s+1}$ since $X$ and $Z$ (and hence $X$ and $Z^{\prime}$ ) are disjoint.

- (2) holds for $T_{s+1}$ by definition of $Z^{\prime}$.

- The argument above shows that (3) holds for $T_{s+1}$ : if $D \in \mathcal{Q}, D \supseteq X$ then $D \cap Z^{\prime}$ is nonempty.

This concludes step $s$ of the construction, and proves the proposition.

4.3. An infinite variant. Another variant of 2IP requires the pairwise intersection of any two sets in a solution to be infinite.

Proposition 4.5 (with R. Kuyper). The following are equivalent for a Turing degree $\mathbf{d}$ :

(1) If $\mathcal{A}$ is a computable instance of an intersection problem then $\mathbf{d}$ computes a subfamily $\mathcal{B}$ of $\mathcal{A}$, maximal with respect to the property "for all $B, C \in \mathcal{B}$, $B \cap C$ is infinite".

(2) $\mathbf{d} \geqslant \mathbf{0}^{\prime \prime}$.

Proof. Since $\mathbf{0}^{\prime \prime}$ can tell whether two elements of $\mathcal{A}$ have infinite intersection or not it can easily build a maximal "infinitary solution".

The direction $(1) \Longrightarrow(2)$ goes in two steps. We first show that we can code $\mathbf{0}^{\prime}$. Define the following family $\mathcal{X}$ : every $X_{e}$ contains a unique identifying marker (say $(0, e))$. Also $(1, s) \in X_{e}$ if and only if $e \notin \varnothing_{s}^{\prime}$. The family $\mathcal{X}$ is computable and has a unique maximal solution, whose index-set $Q$ is the complement of $\varnothing^{\prime}$. Thus a degree satisfying (1) enumerates $Q$ and so computes $\varnothing^{\prime}$.

Next we compute $\varnothing^{\prime \prime}$ with the aid of $\varnothing^{\prime}$. We define a family $\mathcal{Y}$. The sets $Y_{e}$ each have unique identifying markers. On top of these, if $e, d<k$ and $s$ is the least stage such that both $\varphi_{e, s}(k) \downarrow$ and $\varphi_{d, s}(k) \downarrow$ then we enumerate $(e, d, k, s)$ into $Y_{d} \cap Y_{e}$. This family is computable. Again it has a unique maximal infinitary solution whose index-set $Q$ is the set of indices of total computable functions. If $\mathbf{d}$ satisfies (1) then $Q$ is $\mathbf{d}$-c.e. This shows that together with $\varnothing^{\prime}, \mathbf{d}$ can compute $\varnothing^{\prime \prime}$.

Note that the proof can be easily modified to deal with the infinitary version of FIP as well. 


\section{REFERENCES}

[DM13] Damir D. Dzhafarov and Carl Mummert. On the strength of the finite intersection principle. Israel J. Math., 196(1):345-361, 2013.

[GM03] Noam Greenberg and Antonio Montalbán. Embedding and coding below a 1-generic degree. Notre Dame J. Formal Logic, 44(4):200-216, 2003.

Department of Mathematics, Victoria University of Wellington, Wellington, New ZEALAND

E-mail address: donwey@msor.vuw.ac.nz

$U R L:$ http://homepages.mcs.vuw.ac.nz/ downey/

Department of Mathematics, Victoria University of Wellington, Wellington, New ZEALAND

E-mail address: greenberg@msor.vuw.ac.nz

URL: http://homepages.mcs.vuw.ac.nz/ greenberg/

Kurt Gödel Research Center, University of Vienna, Vienna, Austria

E-mail address: turetsd4@univie.ac.at

$U R L:$ http://tinyurl.com/dturetsky 